\title{
Evaluation of Different Surgical Techniques for the Management of Bovine Obstructive Urolithiasis
}

Parrah JD, Moulvi BA, Mohsin Ali Gazi*, Makhdoomi DM, Athar H, Hamadani H and Khan QA

Division of Veterinary Surgery and Radiology, Faculty of Veterinary Sciences and Animal Husbandry, Sher-e-Kashmir University of Agricultural Sciences and Technology of Kashmir, India

\begin{abstract}
Thirty clinical cases of obstructive urolithiasis in cattle calves were managed by three surgical techniques i.e., tube cystostomy with polyvinyl chloride catheter (group Al), tube cystostomy with Foley's catheter (group All) and cystostomy with indwelling urethral catheterization (group B). All the animals were given litholytic agents, antiinflammatory drugs, antibiotics and urine acidifiers postoperatively. These surgical techniques were evaluated on the basis of time taken for each kind of surgery, initiation/free flow of urine, removal of catheters following free flow urination, postoperative complications and overall success rate. Tube cystostomy with Foley's catheter was found the quickest and easiest technique. The median time of onset of free flow of urine from the external urethral orifice in the animals of group Al and All was 9 days with the range of 4-12 and 5-13 days respectively. The main postoperative complications recorded include: catheter dislodgement (one animal each in all the groups), catheter loss (one animal each in group $\mathrm{Al}$ and $\mathrm{B}$ ), catheter blockade (one animal each in group $\mathrm{Al}$ and $\mathrm{B}$ and 3 animals in group All), and urethral rupture (one animal in group Al and 2 animals in group All). The survival rate was higher in the animals of group $A$; however the recovery rate without post-operative complications was higher in the animals of group B.
\end{abstract}

Keywords: Indwelling urethral catheterization; Tube cystostomy; Urolithiasis

\section{Introduction}

Urolithiasis in India presents an important economic repercussion where cattle-based agriculture is strongly linked with the livelihood of an important segment of the population. In India, urolithiais has been commonly reported in bullocks [1], while in Kashmir valley urolithiasis is most prevalent among cow calves below 1 year of age [2]. Treatment of obstructive urolithiasis has been found to vary depending upon clinical status of the animal and duration of obstruction $[3,4]$. Medical treatment has been described with marginal success in relieving the obstruction during early stages of the disease [5]. However, once urethral obstruction is complete, surgical intervention becomes warranted [6,7]. The different surgical interventions employed for the management of obstructive urolithiasis in cattle are aimed either at urolith removal for normal urine flow establishment or for urinary diversion to allow the time for the urinary tract to restore patency. The choice of procedure depends on the extent of tissue damage secondary to the obstruction, the value of the animal, and the owner's expectations for continued use of the animal [8]. Different surgical interventions envisaging urethral obstruction removal for establishment of normal urine flow and urinary diversion techniques, in conjugation with supportive treatments like peritoneal lavage, urine acidifiers and urinary antiseptics, are employed for the management of urethral obstruction in cattle. The surgical techniques include penile transaction with urethral fistulation [9], cystic catheterization [10], pelvic urethrotomy [11], percutaneous tube cystostomy [12] and bladder marsupialization [13]. Perineal urethrotomy and urethrostomy techniques have poor long-term outcome, because of stricture formation of urethrotomy/urethrostomy site, which leads to repeat urethral obstruction [6]. Urinary diversion techniques (ante - pubic urethrostomy) are unsuitable for breeding animals [6,14]. Bladder marsupialisation has been associated with extensive urine scalding problems; stoma stricture and bladder prolapse through the fistula site $[13,15]$.

Recurrent urolithiasis, calculi at multiple sites, badly damaged urethra, atonic bladder or severe cystitis are the common complications that may ensue in failure of surgical management of obstructive urolithiasis. Tube cystostomy, documented by earlier researchers [16] and cystostomy with indwelling urethral catheterisation provide an alternative surgical technique in the management of obstructive urolithiasis. Tube cystostomy though reportedly successful in small ruminants [17], yet the scanned literature indicates its lack of application in large ruminants especially in cattle. Cystostomy with indwelling urethral catheterisation occasionally being performed in individual cases at this institute has not been evaluated in a controlled study and neither is depicted in literature. Therefore, these surgical procedures can form alternative techniques for the management of obstructive urolithiaisis. Being in infancy stages, these techniques need to be evaluated in more number of clinical cases before their recommendation for routine field use. Thus keeping in view the high incidence, heavy economic losses, high treatment and management cost, the present study was undertaken to develop an economical and easy technique for the management of obstructive urolithiasis in cattle. Besides an attempt was made to simplify the conventional tube cystostomy by replacing the Foleys catheter with simple polyvinyl chloride tubing for smooth application in the field.

\section{Materials and Methods}

Thirty male cattle calves, suffering from complete retention of

*Corresponding author: Mohsin Ali Gazi, Division of Veterinary Surgery and Radiology, Faculty of Veterinary Sciences and Animal Husbandry, Sher-e-Kashmir University of Agricultural Sciences and Technology of Kashmir, India, Tel: 0191 2262134-133, extn 13; E-mail: mohsingazi9975@gmail.com

Received March 04, 2014; Accepted November 25, 2014; Published November 28, 2014

Citation: Parrah JD, Moulvi BA, Ali Gazi M, Makhdoomi DM, Athar H, et al (2014) Evaluation of Different Surgical Techniques for the Management of Bovine Obstructive Urolithiasis J Veterinar Sci Technol 5: 203. doi:10.4172/2157 7579.1000203

Copyright: $\odot 2014$ Parrah JD, et al. This is an open-access article distributed under the terms of the Creative Commons Attribution License, which permits unrestricted use, distribution, and reproduction in any medium, provided the original author and source are credited. 
urine, presented for treatment at Teaching Veterinary Clinical Services Complex, Faculty of Veterinary Sciences and Animal Husbandry (F. V. Sc \& A. H.), Sher-e-Kashmir University of Agricultural Sciences and Technology of Kashmir (SKUAST-K), Srinagar, formed the material of the study. These animals were subjected to ultrasonographic examinations for confirmation of the tentative diagnosis, and to know the severity of the condition. For ultrasonographic examination animals were restrained in dorsal recumbency without any sedation. Ventral abdomen was shaved, cleaned with detergent soap and degreased with alcohol. A copious amount of gel was applied. For transabdominal scanning of urinary bladder and percutaneous scanning of penile urethra, low frequency $(3.5 \mathrm{MHz})$ and high frequency $(7.5 \mathrm{MHz})$ transducers attached with a real time; B-mode diagnostic ultrasound scanner (Larson and Tobro) were used respectively. Ten bovine clinical cases of obstructive urolithiasis each were subjected to tube cystostomy using polyvinylchloride urinary catheter (group AI), tube cystostomy using Foley's catheter (group AII) and cystotomy with normograde cystourethral catheterization \{(cystotomy with indwelling urethral catheterization) (group B)\}. Preoperatively fluid and supportive therapy was given to animals with severe dehydration and/or uraemia as per the requirement of the case. All the animals were operated under same anaesthetic technique i.e. local infiltration of left paramedian area starting from the rudimentary teats. Cystorraphy was performed in all the animals after necessary debridement. In intact urinary bladder cases, cystotomy was performed for retrieval of majority of uroliths for further analysis and then cystorraphy was performed in all the animals after necessary debridement, wherever necessary.

For tube cystostomy with polyvinylchloride tubing (group AI) one $\mathrm{cm}$ stab incision was made on the craniovental aspect of the bladder through a pre-placed purse string suture. A fenestrated polyvinylchloride catheter was passed through this incision and preplaced purse string suture was tightened (Figure 1A). The fixation of catheter was further reinforced by applying a Lamberts suture through the catheter. For creating a subcutaneous tunnel a nick in the skin was given with a BP blade at the intended site of the catheter outlet near the prepucial orifice. A straight mosquito forceps was passed through this incision subcutaneously parallel to the laparocystotomy incision till it reached the level of catheter inlet into the bladder. Again a nick with BP blade in the abdominal muscles from inside the abdominal cavity was made for the mosquito forceps to pass through. The jaws of the forceps inside the abdominal cavity were opened and the free end of the catheter was grasped (Figure 1B) and was anchored with the skin (Figure 1C). However, in group AII animals Foley's catheter was introduced into the abdominal cavity from outside inwards across the already created subcutaneous tunnel. The Foley's catheter was introduced into bladder lumen through the stab incision and its bulb was inflated with normal saline for fixation. The Foley's catheter was sutured at multiple sites on the ventral abdomen (Figure 1D-1F).

For performing cystotomy with indwelling urethral catheterisation in the animals of group B, the urinary bladder was exposed through left paramedian approach. In case of ruptured bladders rent in the urinary bladder was freshened and enlarged wherever necessary for retrieval of uroliths and passage of polyvinyl chloride catheter of appropriate size in a normograde manner through the bladder neck. In intact bladders $2 \mathrm{~cm}$ incision was given on the ventral side of the body of the urinary bladder. A sterilized scooter clutch wire was inserted through the catheter to increase its rigidity and act as a stylet (Figure 2A) with the application of moderate force the catheter was passed through the bladder neck into the urethra and out through the prepucial orifice (Figure 2B and 2C), where it was anchored with skin. However urethrotomy's were
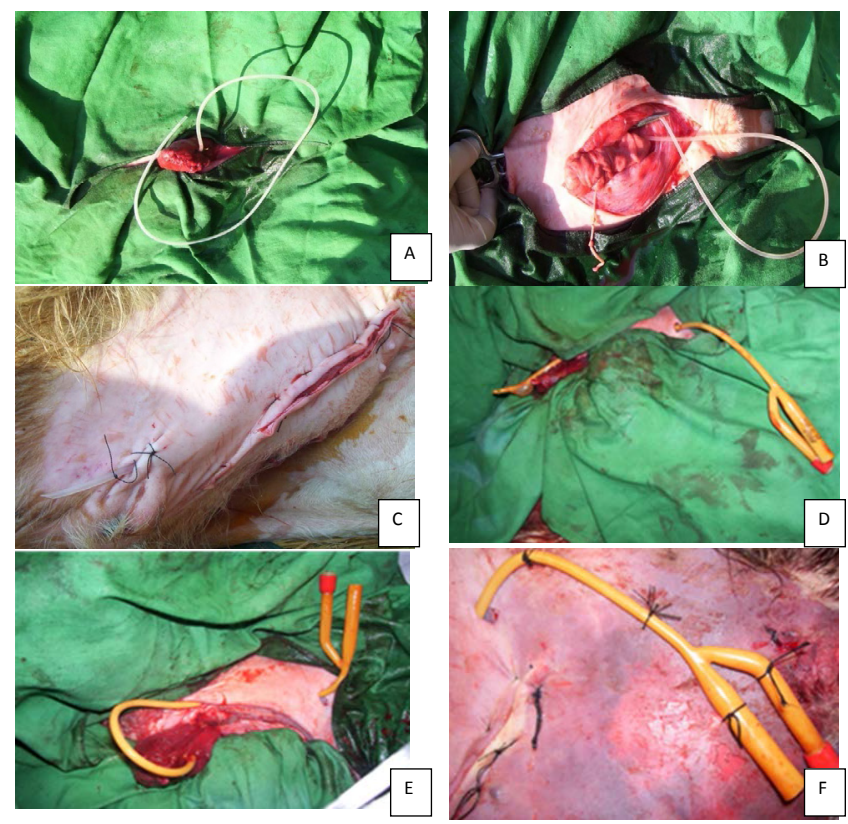

Figure 1: (A) Fixation of simple tube cystostomy catheter into the urinary bladder. (B) Retrieval of simple tube cystostomy catheter out through the subcutaneous tunnel. (C) Simple tube cystosomy catheter out of the subcutaneous tunnel near the prepucial orifice and anchored with skin. (D) Introduction of Foleys catheter through the subcutaneous tunnel. (E) Fixation of Foleys catheter into the urinary bladder. (F) Foleys catheter anchored with skin.
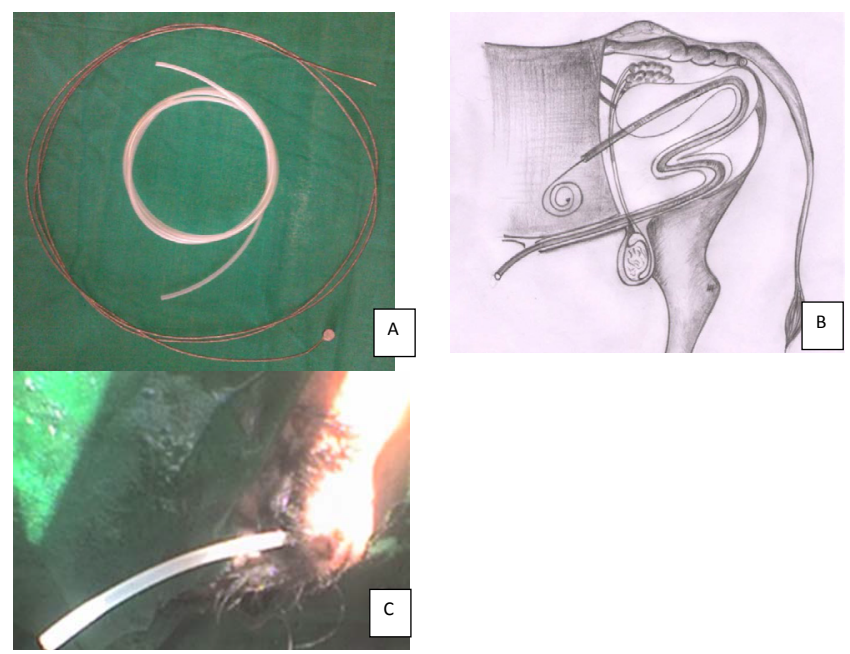

Figure 2: (A) Scooter clutch wire and polyvinyl chloride tubing. (B) Sketch depicting passage of indwelling urethral catheter. (C) Indwelling urethra catheter out of prepucial orifice.

performed for removal of obstructing calculi in cases where catheter could not cross the site of obstruction. Laparotomy and urethrotomy incisions ware closed as per routine procedures.

Initially broad spectrum antibiotics were given; later on antibiotics were given as per the results of antibiotic sensitivity tests performed on the urine samples obtained during operation under strict aseptic conditions in sterile vials. Anti-inflammatory/analgesics were given for five days post operatively. Herbal litholytic drug Tablet Cystone (Herbal Remedies Bangalore India Ltd.) was advised at 2 tablets twice 
Citation: Parrah JD, Moulvi BA, Ali Gazi M, Makhdoomi DM, Athar H, et al. (2014) Evaluation of Different Surgical Techniques for the Management of Bovine Obstructive Urolithiasis. J Veterinar Sci Technol 5: 203. doi:10.4172/2157-7579.1000203

Page 3 of 6

a day for 15 days. Ammonium chloride at $8 \mathrm{gm} / \mathrm{animal}$ was given for acidification of urine. Owners were advised to add sodium chloride to the drinking water at $4 \%$ to enhance frequent water intake and aid in acidification of urine. Acidifiers were given on the basis of the results obtained during previous studies conducted under identical conditions, wherein cent percent cases, phosphate calculi were retrieved which formed in alkaline medium after feeding all concentrate diet to calves $[18,19]$. Surgical wounds were dressed aseptically on alternate days for periods of 10 days or till the sutures were removed.

Duration of surgery was recorded from skin incision to completion of skin sutures. The total time taken in each case was recorded and compared between the groups. Cystostomy tube was plugged/clamped for one hour daily after first operative day to determine if the urethra had become patent. If signs of discomfort (repetitive posturing to urinate, stranguria) were observed, the plug or tie was removed, and the animal allowed urinating through the catheter. The time at which dribbling of urine and normal urination took place was recorded for each case in the animals of groups AI and AII.

Problems encountered, if any, right from the restraining till completion of the surgery were recorded. The animals were kept under close observation for recording any kind of post-operative complications, if encountered, and for their survivability. In case of any mortality, the animal was subjected to full necropsical examination.

The data thus obtained was classified and subjected to statistical analysis as per the standard procedures [20] and inferences drawn.

\section{Results and Discussions}

\section{Ultrasonographic confirmation of diagnosis}

Ultrasound offers a non-invasive method for diagnosis of urolithiasis, localisation of urethral calculi, as well as diagnosis of dilated urethra, cystitis, urethritis and rupture of urethra or the urinary bladder [21]. Ultrasound is ideally suited for examination of urinary bladder, as small bladder cannot be detected by abdominal palpation or radiography [22]. The tentative diagnosis of obstructive urolithiasis reached at clinical examination was confirmed mainly on ultrasound examination. Obstructive urolithiasis with intact urinary bladders was depicted on sonograms as anechoic fluid surrounded by hyperechoic urinary bladder wall with no fluid depiction in the peritoneal cavity (Figure 3A). Ruptured urinary bladders were diagnosed by clear rents in cystic wall and fluid depiction in peritoneal cavity (Figure 3B). Urinary bladder rupture is suspected whenever there is accumulation of urine into the peritoneal cavity and little urine in the bladder lumen. Bladder rupture is also suspected when it is not visible on ultrasound examination [23].

Cystoliths were observed as multiple small hyperechoic structures of varying size swirling in the anechoic fluid (urine) without any acoustic shadows; however in three cases, single calculus found freely floating in the urinary bladders with hyperechogenesity and acoustic shadowing (Figure 3C). Likewise urethrouroliths were diagnosed as hyperechoic masses in the anechoic medium (Figure 3D). The hyperechoic structure showing acoustic shadow is a confirmatory feature of the calculi in the kidneys, urinary bladder or urethra [24]. In rams suffering from obstructive urolithaisis, urinary calculi have been depicted as hyperechoic material with acoustic shadow on ultrasonographic examination [25]. Small calculi do not always produce distal acoustic shadow when they are smaller than the active element diameter of the transducer or calculi are not in the focal zone [26].

\section{Duration of surgery}

The median time of completion of surgical procedure was 40,31 and 85 minutes in groups AI, AII and B respectively (Table 1). The median time taken for completion of surgery in the animal of group $\mathrm{B}$ was highest, which could be attributed to the time consuming procedures of normograde cystourethral catheterisation and suturing of urethral incisions. The operative procedure for tube cystostomy with simple catheters was more time consuming than that for the tube cystostomy with Foleys catheter. This could be attribute to the fact that the simple polyvinylchloride catheter needs to be fixed in the cystic lumen and with the body wall, while no such procedure of anchorage is required for Foleys catheter in the cystic lumen and simple 2 or 3 sutures are required for fixing the Foleys catheter with abdominal wall. In all the groups, the median time for completion of surgical procedures in ruptured urinary bladder cases was more than that in intact urinary bladder cases. This could be attributed to the fact that in ruptured urinary bladder cases, debridement procedures at rent sites and suturing of bladder at the inaccessible neck site were more time consuming. On the basis of time required for the different techniques, tube cystostomy with Foleys catheter was found to be least time consuming and cystotomy with indwelling urethral catheterisation was most time consuming procedure.

\section{Time taken for normal urination}

The median time of initiation of dribbling of urine in the animals of group AI and AII treated by tube cystostomy was 7 and 6 days respectively, and initiation of free flow of urine through external
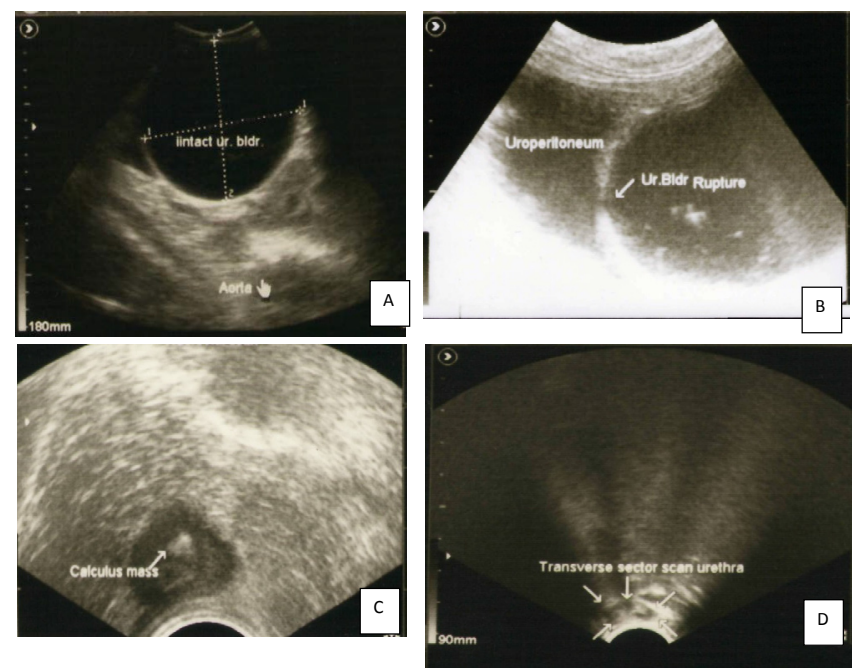

Figure 3: (A) Sonogram showing intact urinary bladder. (B) Sonogram showing ruptured urinary bladder. (C) Sonogram showing calculus mass in urinary bladder. (D) Sonogram showing urethrourolith.

\begin{tabular}{|l|c|c|c|c|c|c|}
\hline \multirow{2}{*}{ Group } & \multicolumn{4}{|c|}{ Time required for completion of surgery (minutes) } \\
\cline { 2 - 7 } & \multicolumn{2}{|c|}{ Whole group } & \multicolumn{2}{c|}{ Intact group } & \multicolumn{2}{c|}{ Ruptured group } \\
\cline { 2 - 7 } & Median & Range & Median & Range & Median & Range \\
\hline Al & $40(10)$ & $30-65(10)$ & $35(3)$ & $35-40(3)$ & $45(7)$ & $30-65(7)$ \\
\hline All & $31(10)$ & $25-55(10)$ & $29(6)$ & $25-35(6)$ & $35(4)$ & $32-55(4)$ \\
\hline B & $85(10)$ & $45-120(10)$ & $85(6)$ & $45-120(6)$ & $80(4)$ & $45-105(4)$ \\
\hline
\end{tabular}

Figures in parenthesis indicate number of animals.

Table 1: Median time required for completion of surgery in different groups of calves suffering from obstructive urolithiasis. 
Citation: Parrah JD, Moulvi BA, Ali Gazi M, Makhdoomi DM, Athar H, et al. (2014) Evaluation of Different Surgical Techniques for the Management of Bovine Obstructive Urolithiasis. J Veterinar Sci Technol 5: 203. doi:10.4172/2157-7579.1000203

Page 4 of 6

urethral orifice was 9 days (Table 2). Mean time of normal urination after tube cystostomy has been recorded as 11 days in previous studies $[17,27]$. The free flow of urine through the external urethral orifice could be due to interplay of many factors. Reduction in inflammation and urethral spasm by administration of anti-inflammatory drugs, drying up of calculi by diversion of urine through the tube cystostomy catheter, dissolution of urethral calculi by acidic urine caused by oral administration of ammonium chloride and of sodium chloride along with drinking water, pulverisation of calculi by litholytic effect of cystone tablets, and occlusion of tube cystotomy catheter helped in achieving urethral patency by flushing the urethra of all debris and calculus material.

In one case of group AI dribbling of urine from the urethral orifice never started and animal died on $15^{\text {th }}$ postoperative day. The animal was subjected to necropsy which revealed severe haemorrhagic urethritis with impacted calculus at the site of sigmoid flexure (Figure 4A). In one case of groups AII free flow of urine never initiated and the animal died
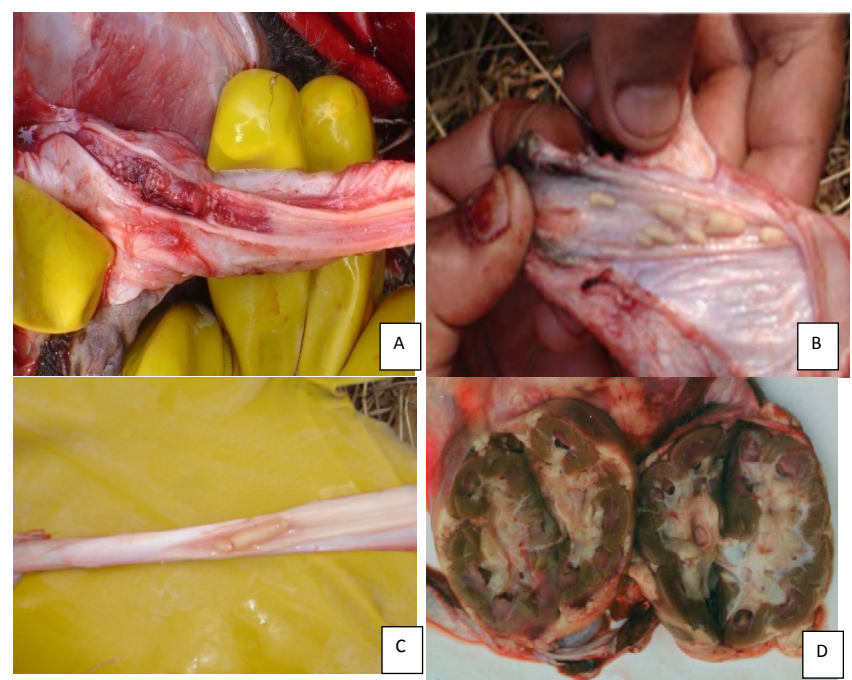

Figure 4: (A) Opened urethra during necropsy showing severe urethritis with impacted calculus. (B) Opened prepuce during necropsy showing numerous loop shaped calculi. (C) Opened urethra during necropsy showing single loop shaped calculus. (D) Opened kidneys during necropsy showing mild hydronephrosis. on 9th postoperative day. On autopsy, prepuce was found packed with multiple calculi (Figure 4B) and the urethra showed single loop shaped calculus in its lumen at the site of sigmoid flexure (Figure 4C). However in both the cases kidneys showed mild hydronephrotic changes (Figure 4D).

Phosphate calculi, mostly recorded in this study because of high grain and low roughage feeding, are formed in alkaline medium $[4,28]$; hence ammonium chloride and sodium chloride induced acidosis helped in the dissolution of calculi. Cystone helped by causing disintegration of calculi by acting on mucin and causing expulsion of calculi by micro pulverisation [29-31] besides its anti-inflammatory action [32]. Occlusion of tube cystostomy catheter also helped in early expulsion of calculi from the urethra by initiating micturation reflexes $[4,27]$.

\section{Time for removal of tube cystostomy and urethral catheter}

The median time for removal of tube cystostomy catheter in group AI, Foley's catheter in group AII and urethral catheter in group B was 11 (10-15), 12 (10-13) and 12 (9-13) days respectively (Table 2). The median time for removal of different types of catheters in different groups was almost same and depended upon the establishment of normal urine flow.

\section{Postoperative complications}

Different postoperative complications recorded in different groups are depicted in Table 2.

Catheter dislodgement and loss: In one animal of group AI catheter was lost on 2nd postoperative day, while in another case the catheter got retrieved back into the abdominal cavity through subcutaneous tunnel. In group AII also Foleys catheter got dislodged in one animal. In group B, two animals had difficulty with catheter. In one case catheter was completely out of the external urethral orifice by second postoperative day, while in another animal the indwelling urethral catheter got dislodged from urinary bladder and its proximal end had reached ischial area of urethra. By 3 rd postoperative day, the catheter was totally out of the external urethral orifice in this animal too.

In this study catheter dislodgement was observed in $2(10 \%)$ animals of tube cystostomy groups. The reason for the dislodgement of Foleys catheter in the animal of group AII could not be ascertained;

\begin{tabular}{|c|c|c|c|c|c|}
\hline S. No. & Observations & Characteristics & Group Al & Group All & Group B \\
\hline \multirow{2}{*}{1.} & \multirow{2}{*}{ Dribbling of urine (Postoperative day) } & Median & 7 & 6 & - \\
\hline & & Range & $3-9$ & $4-9$ & - \\
\hline \multirow{2}{*}{2.} & \multirow{2}{*}{ Free flow of urine (Postoperative day) } & Median & 9 & 9 & - \\
\hline & & Range & $4-12$ & $5-13$ & - \\
\hline \multirow{2}{*}{3.} & \multirow{2}{*}{ Removal of catheter (Postoperative day) } & Median & 11 & 12 & 12 \\
\hline & & Range & $10-15$ & $10-13$ & $9-13$ \\
\hline 4. & \multicolumn{5}{|c|}{ Post-operative complications } \\
\hline 4.1 & Catheter loss & No. of cases & 1 & - & 1 \\
\hline 4.2 & Catheter dislodgement & No. of cases & 1 & 1 & 1 \\
\hline 4.3 & Catheter blockade & No. of cases & 1 & 3 & 1 \\
\hline 4.4 & Urethral rupture & No. of cases & 1 & 2 & 0 \\
\hline 4.5 & Requirement of second surgical intervention & No. of cases & 3 & 3 & 1 \\
\hline \multirow{3}{*}{5.} & \multirow{3}{*}{ Outcome (Survivability) (No. of cases) } & Recovery without any complication & 3 & 6 & 7 \\
\hline & & Recovery with complication & 6 & 3 & 0 \\
\hline & & Deaths & 1 & 1 & 3 \\
\hline
\end{tabular}

Table 2: Postoperative observations and complications recorded in the animals of different groups. 
however deflation of bulb of the Foleys catheter and break of anchoring stitches could be incriminated as the probable cause of its dislodgement. In earlier studies dislodgement of Foleys catheter has been reported in 5 out of $10(50 \%)$ goats treated by tube cystostomy [23]. In one animal of group AI catheter got retrieved back into abdominal cavity. Retrieval of tube cystostomy catheter back into abdominal cavity through subcutaneous tunnel could be due to break of anchoring stitch of tube cystostomy catheter with skin and pulling force applied to catheter by contracting urinary bladder. In one animal of group AI, tube cystostomy catheter was removed by animal itself. In group B catheter dislodgement and loss was observed in $2(20 \%)$ animals. In one animal, catheter dislodgement was observed following urethral leakage at the ischial urethrotomy site. Dislodgement of urethral catheter followed by leakage of urine has also been reported in previous studies as one of the complications of urethrotomy [24-26]. In second animal of group $\mathrm{B}$, no urethrotomy was performed during urethral catheterisation; the removal of indwelling urethral catheter could be attributed to forceful micturation efforts as the animal passed urine normally after dislodgement of the catheter.

Catheter blockade: The occurrence of tube cystostomy catheter blockade was less in group AI than in group AII, which could be attributed to fenestration of polyvinylchloride tube cystostomy catheter as compared to single point exit in the Foleys catheter. In one animal of group AI and in three animals of group AII catheters got blocked by 3rd postoperative day and were relieved by flushing with normal saline solution. In one animal of group B indwelling urethral catheter got blocked by 7 th postoperative day and blockade could not be relieved by flushing with normal saline solution. The blockade might have occurred by urinary sludge, blood clots, sandy material left in urinary bladder, and mucosal shreds. Blockade of Foley's catheter with blood has been recorded in previous studies too [27]. Failure to remove the blockade in the urethral catheter by flushing could be due to its kinking, as blockade and kinking of urethral catheter has also been reported by previous researchers [4].

Urethral rupture: Urethral rupture, characterised by subcutaneous accumulation of urine on ventral abdomen, as a postoperative complication was recorded in one animal of group AI and 2 animals of group AII. No urethral rupture was recorded in any animal of the group B. All the animals had the complaint of blockade of tube cystostomy catheters before rupture of urethra. The blockade of catheter probably caused the accumulation of urine in urinary bladder and created pressure over the lodged calculi. This pressure dislodges the calculi but sometimes causes the perforation in weak and necrosed urethra. Rupture of urethra has also been reported as one of the complications of tube cystostomy in small ruminants $[4,27,33]$.

Urine leakage from urethrotomy site (group B): Urinary leakage from urethrotomy site was observed in one animal of group B following initial partial and then complete blockade of urethral catheter on 7th postoperative day. Blockade most probably due to kinking of the catheter, as it could not be cleared by flushing, made the urine to flow around the outside of the catheter and also around the repaired urethrotomy site and lead to leakage of urine [4].

Requirement of second surgical intervention: Second surgical intervention was made in total 7 animals, 3 each in group AI and AII, and one in group B. Second tube cystostomy was performed, one each in the animals of group AI and AII, after complete dislodgement of tube cystostomy catheters, and one in group B following corrective flushing refractory urethral catheter blockade (urethral kinking). The reason for choosing 2 nd tube cystostomy procedure was to maintain the diversion of urine. The procedure, being simple, is advocated in the complicated cases [32]. Second laparotomy was performed to bring back tube cystostomy catheter to the outside of the abdominal cavity through the subcutaneous tunnel. No such retrieval of Foley' catheter is possible because of its branched shape and its anchoring with skin at multiple sites. Multiple nicks on either side of the linea alba were made in the cases of secondary urethral rupture to remove out accumulated urine from the ventral abdominal wall concomitantly with flushing the tube cystostomy catheters to ensure the diversion of urine. These cases recovered without any further complications. Rupture of urethra has been treated successfully by tube cystostomy in a goat [34-36].

Recovery/Success rate (Survivability): The overall success rate was $83.33 \%(25 / 30)$. The survival rate was $90 \%$ each in group AI and AII. The least survival rate was recorded in group B. A higher success rate with tube cystostomy could be attributed to its simplicity. The findings are in total agreement with those of previous researchers [17], who treated 12 out of $14(85.74 \%)$ animals successfully with tube cystostomy. The overall recovery rate without any complication was $64 \%(16 / 25)$. All the surviving animals of group B (7/10), though least among all the groups, recovered without any complications. This could be attributed to the fact that during indwelling urethral catheterisation all the obstructing calculi in the lower urinary tract are expelled out, while in tube cystostomy urethral calculi are left undisturbed and allowed to be dried, pulverised and to be expelled after urethral spasm and inflammation is overcome. This could be the reason for lower rate of normal recovery without any complication in the animals of group AI 3/9 (33.33\%) and group AII 6/9 (66.66\%).

\section{Conclusion}

On the basis of the study it could be concluded that the tube cystostomy with simple polyvinyl chloride tubing is simple and cheap. Cystotomy with indwelling urethral catheterisation, though time consuming, little bit cumbersome and having least survival rate is inherited with least complications. Cystotomy with indwelling urethral catheterisation, requiring simple tubing and scooter clutch wire, should be attempted at first in every clinical case; if successful it will flush out all the calculi from the urethral lumen. With the gain of experience and innovations, this technique could be further simplified for ready to use in field conditions.

\section{References}

1. Ashturkar RW (1994) Urolithgiasis in bullocks-Review of twenty three cases Ind Vet J 71: 489-492.

2. Parrah JD, Moulvi BA, Hussain SS, Sheik GM (2010) Occurrence of obstructive urolithiasis in cattle of Kashmir. SKUAST J Res 12: 193-199.

3. Larson BL (1996) Identifying, treating and preventing bovine urolithiasis. Vet Med 91: 366-377.

4. Van Metre D (2004) Urolithiasis Farm Animal Surgery, Eds Susan L. Fubini and Norm G. Ducharme, W. B. Saunders, New York, 534-547.

5. Crookshank HR (1970) Effect of ammonium salts on the production of ovine urinary calculi. J Anim Sci 30: 1002-1004.

6. Haven ML, Bowman KF, Engelbert TA, Blikslager AT (1993) Surgica management of urolithiasis in small ruminants. Cornell Vet 83: 47-55.

7. House JK, Smith BP, George LW (1996) Obstructive urolithiasis in ruminants Medical treatment and urethral surgery. Comp Cont Edu Pract Vet 18: 317-328.

8. Wolfe DF (1998) Urolithiasis. In: Wolfe DF, Moll HD (eds.) Large Animal Urogenital Surgery. Williams and Wilkins USA. 349-354.

9. Misk NA, Semieka MA (2003) Clinical studies on obstructive urolithiasis in male cattle and buffalo. Assuit Vet Med J 49: 258-274. 
Citation: Parrah JD, Moulvi BA, Ali Gazi M, Makhdoomi DM, Athar H, et al. (2014) Evaluation of Different Surgical Techniques for the Management of Bovine Obstructive Urolithiasis. J Veterinar Sci Technol 5: 203. doi:10.4172/2157-7579.1000203

10. Hussain SS, Moulvi BA (1986) Treatment of ruptured urinary bladder in a male calf: A case report. Ind Vet J 63: 948-951.

11. Ravikumar SB, Shridhar WB (2003) Pelvic urethrotomy through infra-anal approach in a bullock- a case report. Ind Vet J 80: 59-60.

12. Streeter RN, Washburn KE, McCauley CT (2002) Percutaneous tube cystostomy and vesicular irrigation for treatment of obstructive urolithiasis in a goat. J Am Vet Med Assoc 221: 546-549, 501.

13. May KA, Moll HD, Wallace LM, Pleasant RS, Howard RD (1998) Urinary bladder marsupialization for treatment of obstructive urolithiasis in male goats. Vet Surg 27: 583-588.

14. Stone WC, Bjorling DE, Trostle SS, Hanson PD, Markel MD (1997) Prepubic urethrostomy for relief of urethral obstruction in a sheep and a goat. J Am Vet Med Assoc 210: 939-941.

15. May KA, Moll HD, Duncan RB, Moon MM, Pleasant RS, et al. (2002) Experimental evaluation of urinary bladder marsupialization in male goats. Vet Surg 31: 251-258.

16. Williams JM, White RAS (1991) Tube cystotomy in dog and cat. J Small Anim Pract 32: 598-602.

17. Ewoldt JM, Anderson DE, Miesner MD, Saville WJ (2006) Short- and longterm outcome and factors predicting survival after surgical tube cystostomy for treatment of obstructive urolithiasis in small ruminants. Vet Surg 35: 417-422.

18. Parrah JD, Hussain SS, Moulvi BA, Singh M, Athar H (2010) Bovine uroliths analysis: A review of 30 cases. Israel J of Vet Med 65: 103-107.

19. Parrah JD, Moulvi BA, Hussain SS, Bilal S, Athar H (2011) Aetiopathogenesis of bovine obstructive urolithiasis. Indian J Anim Res 45: 1-13.

20. Snedecor GW, Cochran WG (1976) Statistical Methods. Iowa State University Press. 20-28, 58-59.

21. Braun $U$ (1993) Ultrasonographic examination of the left kidney, the urinary bladder, and the urethra in cows. Zentralbl Veterinarmed A 40: 1-9.

22. Biller DS, Kantrowitz B, Partington BP (1990) Diagnostic ultrasound of the urinary bladder. J Am Anim Hosp Assoc 26: 397-402.
23. Braun U, Nuss K, Wapf P, Lischer C (2006) Clinical and ultrasonographic findings in five cows with a ruptured urachal remnant. Vet Rec 159: 780-782.

24. Saini NS, Singh SS (2002) Ultrasonographic and radiographic diagnosis of renal calculi in dogs. Indian J Vet Surg 23: 55

25. Braun U, Schefer U, Föhn J (1992) Urinary tract ultrasonography in normal rams and in rams with obstructive urolithiasis. Can Vet J 33: 654-659.

26. Magda A (2006) Diagnosis of obstructive urolithiasis in cattle and buffalo by using ultrasonography. Online J Vet Res 10: 26-30.

27. Rakestraw PC, Fubini SL, Gilbert RO, Ward JO (1995) Tube cystostomy fo treatment of obstructive urolithiasis in small ruminants. Vet Surg 24: 498-505.

28. Smith LH (1994) Dietary management of urolithiasis. Curr Opin Nephro Hypertens 3: 189-194.

29. Singh NB (1980) Conservative management of ureteric calculus. Probe 3: 204

30. Chatterjee BN (1982) Role of cystone in various urinary disorders. Probe 1: 27

31. Misger MS (1982) Controlled trial in 100 cases with nephro- uretero-lithiasis by cystone. Current Med. Pract 26: 327.

32. Singh PP (1983) Indigenous drugs in modern medicine: A study on cystone. Archives Med Pract 1: 43.

33. Fortier LA, Gregg AJ, Erb HN, Fubini SL (2004) Caprine obstructive urolithiasis: requirement for 2 nd surgical intervention and mortality after percutaneous tube cystostomy, surgical tube cystostomy, or urinary bladder marsupialization. Ve Surg 33: 661-667.

34. Gera KL, Nigam JM (1979) Urolithiasis in bovines (a report of 193 clinical cases). Ind Vet J 56: 417-423.

35. Walker DF, Hull BL (1984) Urinary system surgery. In: Jennings PB (Eds) The practice of large animal surgery, 2: 1072-1080.

36. Pearce SG, Dearo AC, Howard BE, Brisson BA (2003) Management of obstructive urolithiasis and concurrent urethral rupture in a goat. Aust Vet $J$ 81: 268-270. 